\title{
SCIENTIFIC REPORTS

\section{OPEN Progressive hyperleukocytosis is a relevant predictive marker for differentiation syndrome, early \\ death, and subsequent relapse in acute promyelocytic leukemia}

Received: 23 November 2018

Accepted: 22 July 2019

Published online: 15 August 2019

\author{
Jae-HoYoon, Hee-Je Kim, Gi June Min, Sung-Soo Park $\mathbb{D}^{\mathbb{D}}$, Young-Woo Jeon $\mathbb{D}^{\mathbb{D}}$, Sung-Eun Lee, \\ Byung-Sik Cho, Ki-Seong Eom, Yoo-Jin Kim, Seok Lee, Chang-Ki Min, Seok-Goo Cho (i) \& \\ Jong Wook Lee
}

Acute promyelocytic leukemia (APL) is generally held to have favorable risk, but we have observed a high incidence of early deaths caused by fatal bleeding and differentiation syndrome (DS). We retrospectively analyzed $259 \mathrm{APL}$ patients from 2002 to 2014 who all received all-trans retinoic acid (ATRA) with the support of sufficient transfusions, followed by 4 days of idarubicin. High-risk status was determined as a diagnostic leukocyte count (WBCdx) $>10 \times 10^{9} / \mathrm{L}$ (Sanz criteria). For patients with hyperleukocytosis, we sometimes conducted leukapheresis and also used hydroxyurea and prophylactic dexamethasone. Because we frequently observed patient fatalities from progressive hyperleukocytosis, we also checked the maximum leukocyte count (WBCmax) and stratified patients by their incremental ratios. The 8 -week cumulative incidence of early death and DS was $13.5 \%$ and $17.8 \%$, respectively. We found that WBCmax correlated better with early death and DS, even in the low-risk group, than WBCdx. Among the patients with WBCdx $<10 \times 10^{9} / \mathrm{L}$, a WBCmax $>43 \times 10^{9} / \mathrm{L}$ correlated with early death (26.7\%) and DS (40.0\%). Also, having a WBCdx of 10 to $43 \times 10^{9} /$ La that increased to a WBCmax $>43 \times 10^{9} / \mathrm{L}$ correlated with increased early death (33.3\%). The multivariate analysis revealed that a WBCmax $>43 \times 10^{9} / L$ correlated significantly with both early death and DS.

Acute promyelocytic leukemia (APL) is generally held to have favorable risk and be related to good hematological remission, with a long-term overall survival (OS) rate of more than $80 \%$ and a lower relapse rate than acute myeloid leukemia ${ }^{1-6}$. The combination of all-trans retinoic acid (ATRA) and anthracycline-based chemotherapy has improved outcomes in APL during the past few decades, and recent trials have shown the safety and efficacy of arsenic trioxide (ATO) and ATRA therapy ${ }^{7-11}$. Nevertheless, we still observe unexpected early deaths from APL, mainly caused by significant bleeding complications, sepsis, or differentiation syndrome (DS), especially in high-risk patients ${ }^{12-15}$.

The National Cancer Institute's Surveillance, Epidemiology, and End Results study analyzed 1,400 patients and reported an early death rate of $17.3 \%$, which was higher than that in previous reports, and that the rate increased with age, especially in patients older than 55 years ${ }^{15}$. For DS, the Programa Español de Tratamientos en Hematología (PETHEMA) group analyzed 739 patients treated with ATRA and anthracycline-based chemotherapy and reported a DS rate of $24.8 \%$; the rate of the severe form of DS was $12.6 \%$, and it was associated with an increase in mortality ${ }^{13}$. However, a wide range of incidence rates have been reported; many clinical trials have excluded patients with early death, and the criteria used for the diagnosis of DS has varied by study.

Sanz-risk scoring is generally used to identify high-risk APL patients, those who present a high leukocyte count at diagnosis. However, we have frequently observed that patients with progressive hyperleukocytosis from low WBCdx to a high maximum leukocyte count (WBCmax) despite attempts to reduce leukocyte counts by

Department of Hematology, Catholic Hematology Hospital and Leukemia Research Institute, Seoul St. Mary's Hospital, College of Medicine, The Catholic University of Korea, Seoul, Korea. Correspondence and requests for materials should be addressed to H.-J.K. (email: cumckim@catholic.ac.kr) 
hydroxyurea, cytarabine, or leukapheresis. Diagnostic and peak leukocyte counts and an abnormal creatinine level were reported as predictive factors for DS ${ }^{13,16}$, but other reports found no significant predictive factors ${ }^{12,17}$.

We designed the current study to identify the incidence and clinical manifestations of early death and DS in patients with APL who were mainly treated with ATRA and anthracycline-based chemotherapy. Then we tried to identify factors associated with the progression of leukocyte counts that might affect the development of DS or early death.

\section{Results}

Baseline characteristics. The main presenting clinical and biological features of all patients (median age: 42 years old [range; 17-72]) at the time of diagnosis are presented in Table 1 . The median leukocyte count at diagnosis was $3.73 \times 10^{9} / \mathrm{L}$ (range; $0.4-172.9$ ), and at peak it was $13.4 \times 10^{9} / \mathrm{L}$ (range; $0.4-177.0$ ). The median platelet count was $32.0 \times 10^{9} / \mathrm{L}$ (range; $5.0-216.0$ ), and the median prothrombin time was $69.0 \%$ (range; $35.0 \%-105.0 \%$ ). Based on Sanz-risk scoring ${ }^{4,13}, 79$ (30.5\%) patients were classified into the high-risk group, 108 (41.7\%) were in the intermediate-risk group, and $72(27.8 \%)$ were in the low-risk group. All patients showed positive PML-RARA by RT-PCR, but 5 patients showed normal karyotype rather than $\mathrm{t}(15 ; 17)$ (q22;q21). Among those with $\mathrm{t}(15 ; 17)$ (q22;q21), additional chromosomal aberrations were observed in 77 (29.7\%) patients. Of the 190 patients with available $B C R$ isotype analyses, $B C R 3$ was identified in $70(36.8 \%)$ patients, and of the 164 patients with available FLT3 mutation analyses, 34 had the FLT3-ITD mutation and 12 had the FLT3-TKD mutation. All but 1 patient received ATRA. Standard chemotherapy using idarubicin and ATRA was administered in 219 (84.6\%) patients, whereas ATO and ATRA was administered in 13 (5.0\%) patients. Patients presenting a severe comorbidity $(\mathrm{n}=26)$ were treated with ATRA alone because ATO was unavailable at that time. Except for 37 patients who died within 8 weeks without post-induction bone marrow (BM) biopsy, $220(84.9 \%)$ patients achieved complete remission (CR) after induction chemotherapy. The remaining 2 achieved CR after reinduction chemotherapy.

Survival outcomes and early events. After a median follow-up of 65.4 months (range; 11.1-170.5) for surviving patients, the 5-year OS and disease free survival (DFS) rates of the entire patient cohort were $76.8 \%$ (Fig. 1A) and 69.8\% (Fig. 1B), respectively. The 5-year non-relapse mortality (NRM) rate of the entire cohort was $1.2 \%$ (Fig. 1C). Among the 222 (86.0\%) patients achieving CR, the 5-year cumulative incidence of relapse (CIR) rate was $17.1 \%$ (Fig. 1D). We defined the time point for early death as 8 weeks, and the 8 -week early death rate was $13.5 \%$ for all patients (Fig. 1E). The causes of early death were hemorrhage in multiple organs $(n=3)$, alveolar hemorrhage $(n=13)$, intracerebral hemorrhage $(n=12)$, severe DS followed by multiorgan failure $(n=4)$, and sepsis $(n=3)$. DS was observed in $46(17.8 \%)$ patients (Fig. 1F), and among them, severe DS was observed in 9 $(19.5 \%)$, of whom 4 died due to respiratory failure and progressive multiorgan failure.

Molecular markers and leukocyte counts. Among the 160 patients with both FLT3 mutation and BCR isotype results available, 45 (28.1\%) were positive for the FLT3 mutation, and 54 (33.7\%) had the BCR3 isotype. Of the 45 FLT3-positive patients, 26 (57.8\%) had the BCR3 isotype, whereas 87 of the 115 (75.7\%) FLT3-negative patients had the BCR1 isotype $(p<0.001)$. Thus, more than half the FLT3-positive patients had the BCR3 isotype, and having either the $B C R 3$ isotype or FLT3-positivity correlated with higher WBCdx (median $9.0 \times 10^{9} / \mathrm{L}$, $p<0.001$ ) and WBCmax (median $23.1 \times 10^{9} / \mathrm{L}, p<0.001$ ) compared to patients with both the BCR1 isotype and FLT3-negativity (median WBCdx $2.1 \times 10^{9} / \mathrm{L}$ and WBCmax $6.1 \times 10^{9} / \mathrm{L}$ ). Patients with both the BCR3 isotype and FLT3-positivity $(\mathrm{n}=26)$ had significantly higher WBCdx (median $\left.23.8 \times 10^{9} / \mathrm{L}\right)$ and WBCmax (median $45.4 \times 10^{9} / \mathrm{L}$ ) than the other patients.

Patients with progressive hyperleukocytosis. According to the Sanz-risk scoring, the leukocyte count cut-off for high-risk patients was $\mathrm{WBCdx}>10 \times 10^{9} / \mathrm{L}$. In a receiver operating characteristic (ROC) curve analysis, we found a significant leukocyte count cut-off for predicting both early death and DS at the level of $>10 \times 10^{9} / \mathrm{L}$ for $\mathrm{WBCdx}$ and $>43 \times 10^{9} / \mathrm{L}$ for WBCmax, respectively. Using those levels, we grouped patients by their leukocyte count increases from their diagnostic levels (Fig. 2). Group analysis using diagnostic leukocyte counts and the increment level was performed except for patients with WBCdx $>43 \times 10^{9} / \mathrm{L}$, who already showed high leukocyte counts. The first leukocyte increment group contained patients with $\mathrm{WBCdx}<10 \times 10^{9} / \mathrm{L}$ $(\mathrm{n}=180)$, and the second group contained patients with WBCdx 10 to $43 \times 10^{9} / \mathrm{L}(\mathrm{n}=47)$. Then, we identified 3 increment subgroups in the $\mathrm{WBCdx}<10 \times 10^{9} / \mathrm{L}$ group - subgroup 1 , sustained low, WBCmax $<10 \times 10^{9} / \mathrm{L}$ (red, $\mathrm{n}=116$ ); subgroup 2, increment to $\mathrm{WBCmax} 10$ to $43 \times 10^{9} / \mathrm{L}$ (pink, $\mathrm{n}=49$ ); subgroup 3, increment to WBCmax $>43 \times 10^{9} / \mathrm{L}$ (red diagonal, $\mathrm{n}=15$ ). Next, we identified 2 subgroups from the WBCdx 10 to $43 \times 10^{9} / \mathrm{L}$ group - subgroup 1, sustained WBCmax 10 to $43 \times 10^{9} / \mathrm{L}$ (blue, $\mathrm{n}=32$ ); subgroup 2 , increment to WBCmax $>43 \times 10^{9} / \mathrm{L}$ (blue diagonal, $\mathrm{n}=15$ ).

Progressive hyperleukocytosis affecting early death and DS. We calculated the cumulative incidence of early death and DS in the 2 large groups and their subgroups. In the low Sanz-risk group - WBCdx $<10 \times 10^{9} / \mathrm{L}$ (Fig. $3 \mathrm{~A}, \mathrm{~B}$ ), the increment to WBCmax 10 to $43 \times 10^{9} / \mathrm{L}$ and increment to WBCmax $>43 \times 10^{9} / \mathrm{L}$ subgroups had increased 8 -week early death rates $(14.3 \%$ and $26.7 \%, p=0.024$ and $p=0.001$, respectively) and DS rates $(24.5 \%$ and $40.0 \%, p=0.005$ and $p<0.001$, respectively) compared with patients with sustained WBCmax $<10 \times 10^{9} / \mathrm{L}$ (3.4\% for early death and $8.6 \%$ for DS). In the WBCdx 10 to $43 \times 10^{9} / \mathrm{L}$ group (Fig. 3C,D), the increment to WBCmax $>43 \times 10^{9} / \mathrm{L}$ subgroup had a higher 8 -week early death rate than patients with sustained WBCmax 10 to $43 \times 10^{9} / \mathrm{L}(33.3 \%$ vs. $3.1 \%, p=0.004)$, but the DS rate did not differ significantly between the two subgroups $(13.3 \%$ vs. $15.6 \%, p=0.800)$.

Maximum leukocyte count (WBCmax) and treatment outcomes. Next, we stratified patients into 4 subgroups according to their maximum leukocyte count (WBCmax). Right bar in the previous Fig. 2 shows the proportion of patients in each subgroup - WBCmax $<10 \times 10^{9} / \mathrm{L}(\mathrm{n}=116)$, WBCmax 10 to $43 \times 10^{9} / \mathrm{L}$ 


\begin{tabular}{|c|c|}
\hline Total $n=259$ & Number or median value \\
\hline Age, median (range) & $42(17-72)$ \\
\hline Gender, Male & $143(55.2 \%)$ \\
\hline \multicolumn{2}{|l|}{ Laboratory findings at diagnosis } \\
\hline Leukocyte count $\left(\times 10^{9} / \mathrm{L}\right)$ Leukocytes count at peak $\left(\times 10^{9} / \mathrm{L}\right)$ & $3.73(0.4-172.9) 13.4(0.4-177.0)$ \\
\hline Hemoglobin $(\mathrm{g} / \mathrm{dL})$ & $8.6(3.8-15.0)$ \\
\hline Platelet $\left(\times 10^{9} / \mathrm{L}\right)$ & $32.0(5.0-216.0)$ \\
\hline Lactate dehydrogenase (LDH, U/L) & $688.0(221.0-5440.0)$ \\
\hline Prothrombin time (PT, \%) & $69.0(35.0-105.0 \%)$ \\
\hline Partial thromboplastin time (aPTT, sec) & $28.0(20-50)$ \\
\hline Fibrinogen $(\mathrm{mg} / \mathrm{dL})$ & $131.0(31.0-685.0)$ \\
\hline Antithrombin III (\%) & $98.0(44.0-150.0)$ \\
\hline $\mathrm{D}$-dimer $(\mathrm{mg} / \mathrm{L})$ & $20.0(1.0-36.0)$ \\
\hline \multicolumn{2}{|l|}{ Sanz-risk criteria } \\
\hline High & $79(30.5 \%)$ \\
\hline Intermediate & $108(41.7 \%)$ \\
\hline Low & $72(27.8 \%)$ \\
\hline \multicolumn{2}{|l|}{ Karyotype } \\
\hline Normal karyotype & $5(1.9 \%)$ \\
\hline $\mathrm{t}(15 ; 17)$ alone & $177(68.4 \%)$ \\
\hline $\mathrm{t}(15 ; 17)$ with 1 additional karyotype & $51(19.7 \%)$ \\
\hline $\mathrm{t}(15 ; 17)$ with $\geq 2$ additional karyotype & $26(10.0 \%)$ \\
\hline \multicolumn{2}{|l|}{$P M L-R A R A$ subtype } \\
\hline Not assessed & $69(26.6 \%)$ \\
\hline BCR1 & $120(46.4 \%)$ \\
\hline BCR3 & $70(27.0 \%)$ \\
\hline \multicolumn{2}{|l|}{ FLT3 mutation } \\
\hline Not assessed & $95(36.7 \%)$ \\
\hline No FLT3 mutation & $118(45.6 \%)$ \\
\hline FLT3-ITD & $34(13.1 \%)$ \\
\hline FLT3-TKD & $12(4.6 \%)$ \\
\hline Leukapheresis at initial treatment & $26(18.2 \%)$ \\
\hline$>3$ times & $6(2.3 \%)$ \\
\hline$\leq 3$ times & $20(15.9 \%)$ \\
\hline \multicolumn{2}{|l|}{ Induction chemotherapy } \\
\hline ATRA alone & $26(10.0 \%)$ \\
\hline ATRA plus idarubicin & $219(84.6 \%)$ \\
\hline ATRA plus arsenic trioxide & $13(5.0 \%)$ \\
\hline Differentiation syndrome & $46(17.9 \%)$ \\
\hline Median onset day (range) & $7.5(1-46)$ \\
\hline \multicolumn{2}{|l|}{ Hematological complete response } \\
\hline After $1^{\text {st }}$ induction & $220(84.9 \%)$ \\
\hline After $2^{\text {nd }}$ induction & $2(0.7 \%)$ \\
\hline
\end{tabular}

Table 1. Baseline characteristics of enrolled patients. Abbreviation: BCR, breakpoint cluster region; FLT3, Fmslike tyrosine kinase 3; ITD, internal tandem duplication; TKD, tyrosine kinase domain; $A B L$, Abelson murine leukemia viral oncogene; WT1, Wilms' tumor 1; ATRA, all-trans retinoic acid; AML, acute myeloid leukemia.

$(\mathrm{n}=81)$, progressed WBCmax $>43 \times 10^{9} / \mathrm{L}(\mathrm{n}=30)$, and sustained WBCmax $>43 \times 10^{9} / \mathrm{L}(\mathrm{n}=32)$. The 8-week early death rates for the 4 subgroups were $3.4 \%, 9.9 \%, 30.0 \%$, and $40.6 \%$, respectively (Fig. $4 \mathrm{~A}$ ), and the DS rates were $8.6 \%, 21.0 \%, 26.7 \%$, and $34.4 \%$, respectively (Fig. $4 \mathrm{~B}$ ). We also calculated the long-term survival outcomes with consideration of the early outcomes. The estimated 5-year OS rates were $98.9 \%$ for the WBCmax $<10 \times 10^{9} / \mathrm{L}$ group, $79.3 \%$ for the WBCmax 10 to $43 \times 10^{9} / \mathrm{L}$ group, $59.3 \%$ for the progressed WBCmax $>43 \times 10^{9} / \mathrm{L}$ group, and $46.0 \%$ for the sustained WBCmax $>43 \times 10^{9} / \mathrm{L}$ group (Fig. $4 \mathrm{C}$ ), and the 5 -year CIR rates were $9.0 \%$ for the $\mathrm{WBC} \max <10 \times 10^{9} / \mathrm{L}$ group, $18.7 \%$ for the WBCmax 10 to $43 \times 10^{9} / \mathrm{L}$ group, $33.5 \%$ for the progressed $\mathrm{WBCmax}>43 \times 10^{9} / \mathrm{L}$ group, and $42.0 \%$ for the sustained $\mathrm{WBCmax}>43 \times 10^{9} / \mathrm{L}$ group (Fig. $\left.4 \mathrm{D}\right)$. The multivariate analysis showed that early death correlated with age older than 40 years $(\mathrm{HR}=2.38,95 \%$ CI: $1.1-5.1, p=0.026)$, progressed $\mathrm{WBCmax}>43 \times 10^{9} / \mathrm{L}(\mathrm{HR}=3.72,95 \% \mathrm{CI}: 1.4-9.8, p=0.008)$, and sustained WBCmax $>43 \times 10^{9} / \mathrm{L}(\mathrm{HR}=5.38,95 \% \mathrm{CI}: 2.2-13.2, p<0.001)$. A higher DS rate correlated with an antithrombin III (ATIII) level $\leq 100 \%(\mathrm{HR}=2.51,95 \% \mathrm{CI}: 1.1-5.9, p=0.036), \mathrm{WBCmax} 10$ to $43 \times 10^{9} / \mathrm{L}(\mathrm{HR}=3.06$, 
A

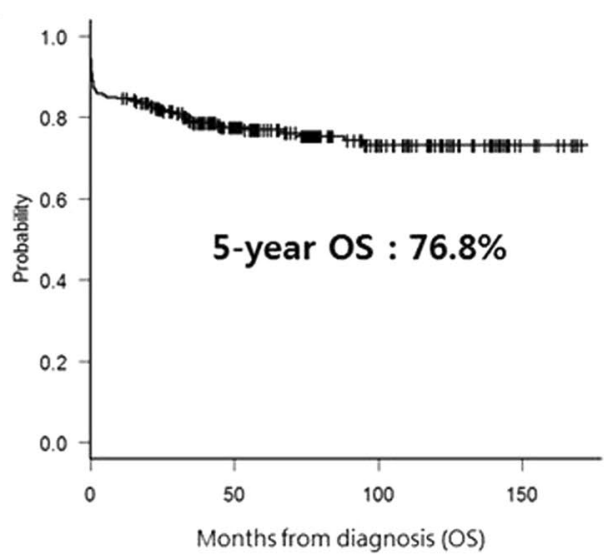

C

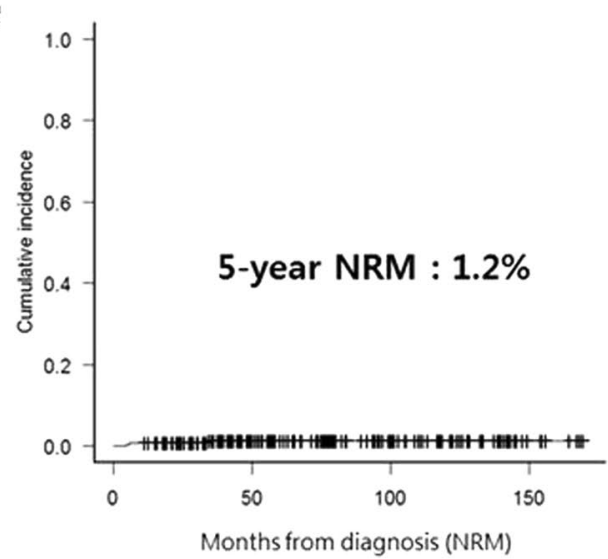

$\mathbf{E}$

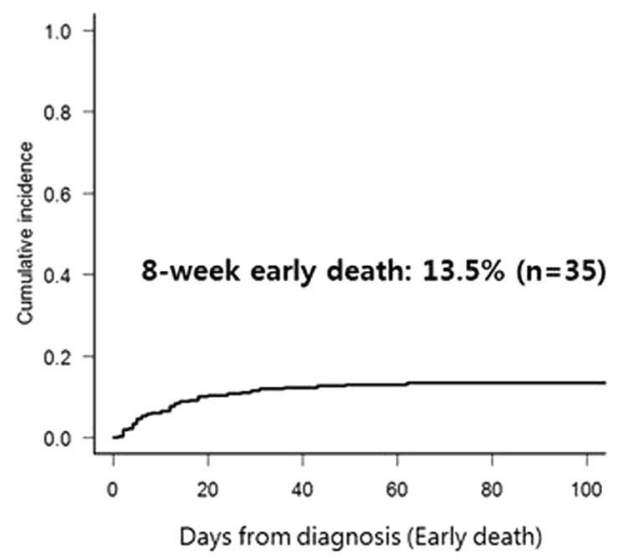

B

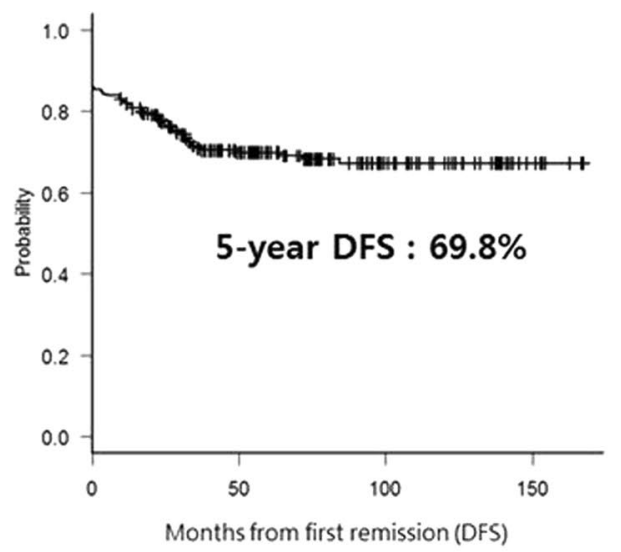

D

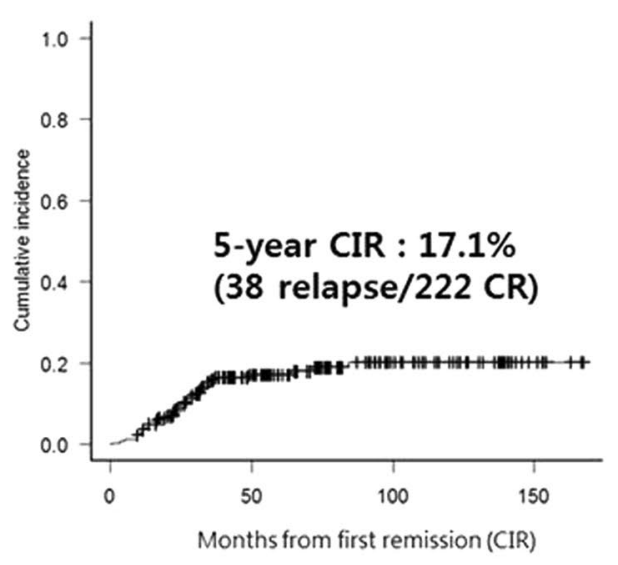

$\mathbf{F}$

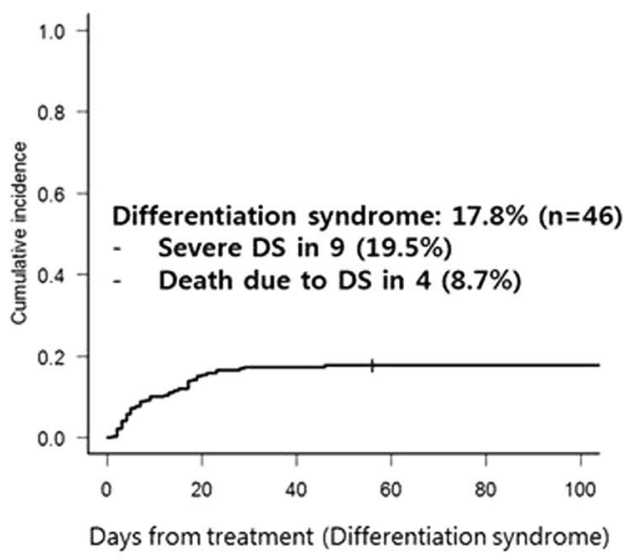

Figure 1. Overall clinical outcomes. (A) OS. (B). DFS. (C) CIR. (D). NRM. (E) Early death (within 8 weeks). (F) DS. Abbreviations; OS, overall survival; DFS, disease free survival; CIR, cumulative incidence of relapse; NRM, non-relapse mortality; DS, differentiation syndrome.

95\% CI: $1.4-6.6, p=0.004)$, progressed $\mathrm{WBCmax}>43 \times 10^{9} / \mathrm{L}(\mathrm{HR}=3.29,95 \% \mathrm{CI}: 1.3-8.2, p=0.011)$, and sustained WBCmax $>43 \times 10^{9} / \mathrm{L}(\mathrm{HR}=5.79,95 \% \mathrm{CI}: 2.5-13.4, p<0.001)$ compared with patients with WBCmax $<10 \times 10^{9} /$ L (Table 2).

\section{Discussion}

In this study, we have found good survival outcomes from our analysis of 259 APL patients treated with ATRA and anthracycline-based chemotherapy based on the AIDA protocol ${ }^{1,18,19}$. As we experienced, except for the 35 (13.5\%) patients who died within 8 weeks, NRM was low, and most long-term failures were caused by APL relapses: 5-year NRM was $1.2 \%$ and 5-year CIR was $17.1 \%$. Most early deaths were caused by significant bleeding complications, including intracranial hemorrhage and alveolar hemorrhage, followed by multiorgan failure.

We also identified the cumulative incidence of DS, which occurred in 46 patients (17.8\%). Of those, severe DS was observed in 9 (19.5\%), and 4 of them died of progressive organ failure caused by the severe DS. This value 


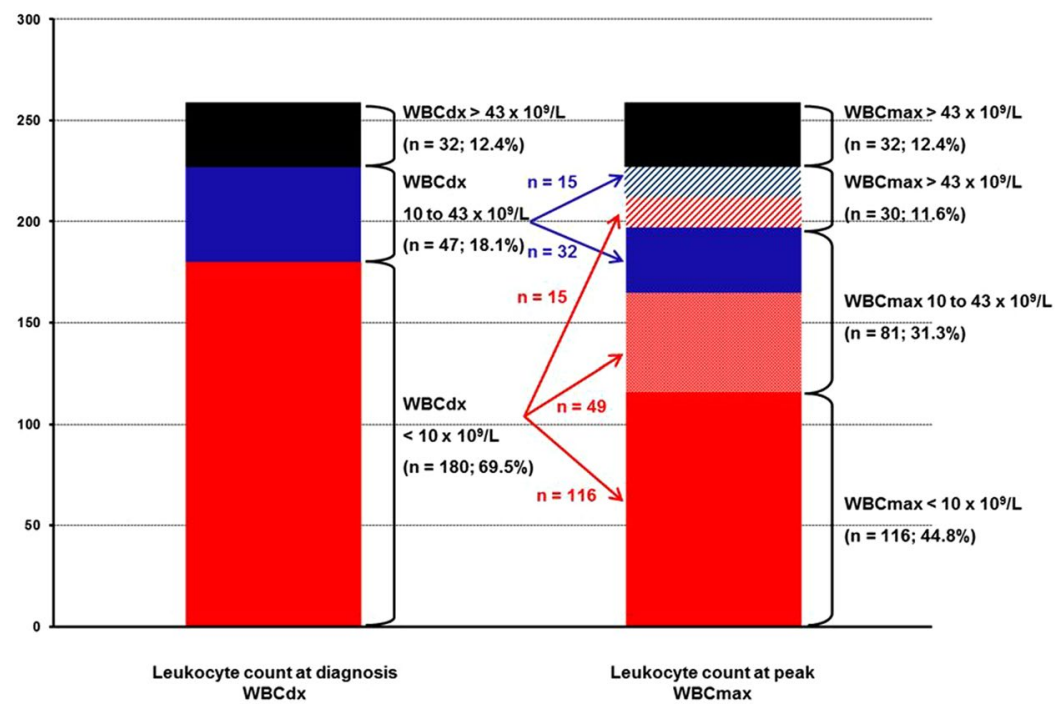

Figure 2. Proportion of patients whose WBC count changed from diagnosis (WBCdx) to peak (WBCmax) during the initial treatment period (right bars). Black bar $(\mathrm{n}=32)$, sustained hyperleukocytosis (both WBCdx and WBCmax $\left.>43 \times 10^{9} / \mathrm{L}\right)$; blue diagonal lines $(\mathrm{n}=15)$, progressive leukocytosis $\left(\mathrm{WBCdx} 10\right.$ to $43 \times 10^{9} / \mathrm{L}$ to $\left.\mathrm{WBCmax}>43 \times 10^{9} / \mathrm{L}\right)$; red diagonal lines $(\mathrm{n}=15)$, progressive leukocytosis $\left(\mathrm{WBCdx}<10 \times 10^{9} / \mathrm{L}\right.$ to WBCmax $\left.>43 \times 10^{9} / \mathrm{L}\right)$; blue bar $(\mathrm{n}=32)$, sustained leukocytosis (both WBCdx and WBCmax 10 to $\left.43 \times 10^{9} / \mathrm{L}\right)$; pink bar $(\mathrm{n}=49)$, progressive leukocytosis $\left(\mathrm{WBCdx}<10 \times 10^{9} / \mathrm{L}\right.$ to $\mathrm{WBCmax} 10$ to $\left.43 \times 10^{9} / \mathrm{L}\right)$; red bar, sustained low WBC (both WBCdx and WBCmax $<10 \times 10^{9} / \mathrm{L}$ ).

is lower than in a previous large cohort $\operatorname{study}^{13}$; most of our DS cases were safely manageable with intravenous dexamethasone and immediate cessation of ATRA. However, we might have missed some patients with severe DS that was misdiagnosed as a bleeding complication, such as alveolar hemorrhage. Of the 35 early deaths in our cohort, 16 patients suffered from alveolar hemorrhage.

Sanz-risk scoring suggests that the leukocyte count prior to therapy is the prognostic factor most relevant to relapse and survival outcomes ${ }^{17,20}$. Although little was previously reported about factors predictive of early death and DS, a study by Vahdat et al. showed that peak leukocyte count correlated with DS ${ }^{16}$, and some studies have suggested that an abnormal creatinine level, male sex, old age, and hyperleukocytosis at diagnosis are related to early death ${ }^{14,17}$. However, no factors have been consistently shown to affect early complications.

We stratified patients into groups by their diagnostic leukocyte count (WBCdx), leukocyte increment ratio, and maximum leukocyte count (WBCmax). Classic Sanz-risk scoring suggests that a WBCdx higher than $10 \times 10^{9} / \mathrm{L}$ is a significant cut-off for long-term outcomes. Our ROC analysis in this study found that both a WBCdx and WBCmax higher than $43 \times 10^{9} / \mathrm{L}$ correlated significantly with early death and DS. Overall, we first identified changing outcomes according to 3 levels of leukocyte increment beginning with a WBCdx lower than $10 \times 10^{9} / \mathrm{L}$. Progression to both WBCmax 10 to $43 \times 10^{9} / \mathrm{L}$ and WBCmax $>43 \times 10^{9} / \mathrm{L}$ correlated with a significantly higher incidence of DS and early death compared with patients with a WBCmax $<10 \times 10^{9} / \mathrm{L}$. In addition, the 2 subgroups with progressive hyperleukocytosis showed significantly higher CIR rates and inferior OS even though they had low leukocyte counts at diagnosis. This might be the first evidence that progressive leukocytosis (as reflected by WBCmax) is a more relevant factor in predicting early and long-term survival outcomes than the leukocyte count prior to therapy. At this point, we question whether ATRA or ATO should be started in patients with hyperleukocytosis and wonder whether cytoreduction or leukapheresis is more important for patients with hyperleukocytosis to reduce the rates of DS and early death. However, no definite guidelines discuss those issues because high-risk APL patients are rare, and individual differences make it difficult to draw definite conclusions for standard management strategies. Nonetheless, the role of prophylactic dexamethasone should be studied in the near future.

The multivariate analysis also revealed that old age correlated with early death, and a low ATIII level correlated with DS. For ATIII, our further analysis revealed that ATIII $>100 \%$ correlated with significantly lower incidence of DS, even in patients with WBCmax $>43 \times 10^{9} / \mathrm{L}$. Because we can support ATIII in patients using disseminated intravascular coagulation and a supportive management strategy, ATIII and transfusions could be adjusted in future prospective trials. In old age, especially for people with comorbidity, intensive chemotherapy might be toxic, causing bleeding complications and sepsis. Therefore, using ATO plus ATRA could be an important alternative for elderly APL patients ${ }^{8,10,11,15}$. A randomized phase III trial demonstrated that ATRA plus ATO is at least non-inferior and could be superior to ATRA plus chemotherapy in low- to intermediate-risk APL ${ }^{8}$. In high-risk APL, a more tailored therapy using ATO and attenuating conventional chemotherapy is needed to reduce the early death rate in elderly patients ${ }^{21}$.

We checked for the FLT3-ITD mutation and break point isoforms (BCR1 and BCR3) in the current study, and we found that the FLT3 mutation and BCR3 isoform correlated with high leukocyte counts and early death. However, that analysis was many missing data points; in the multivariate analysis, no molecular parameter 
A

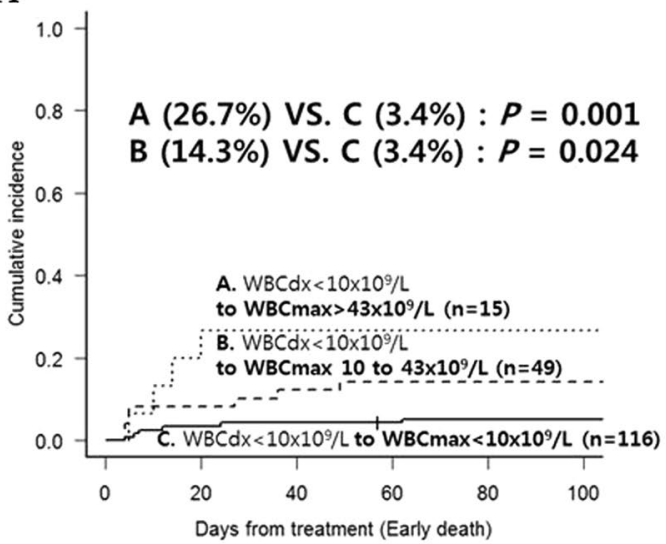

C

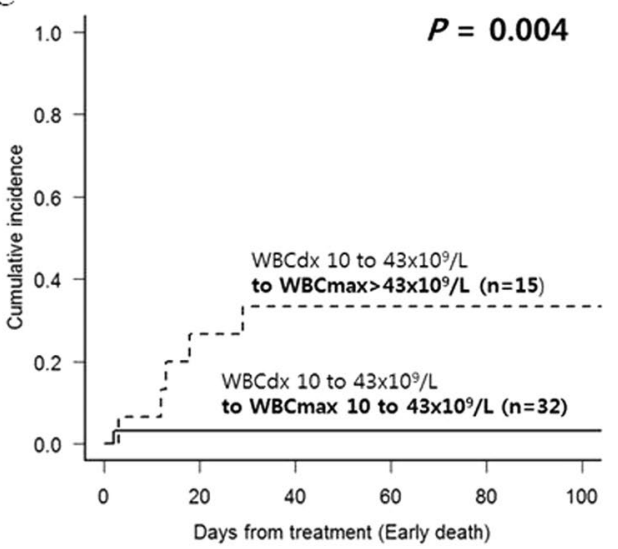

B

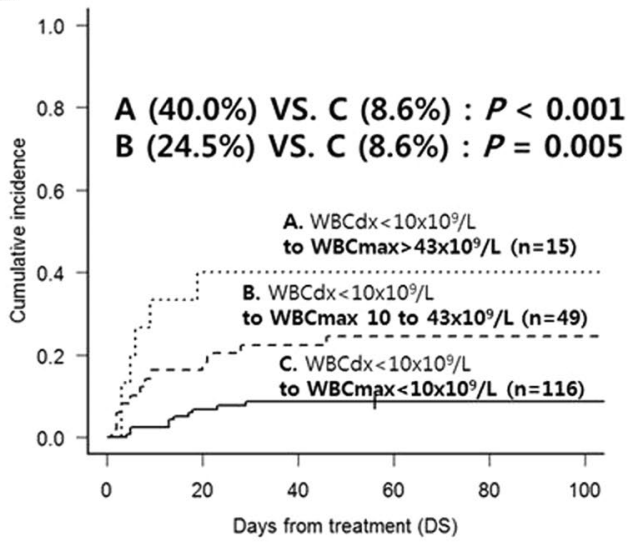

D

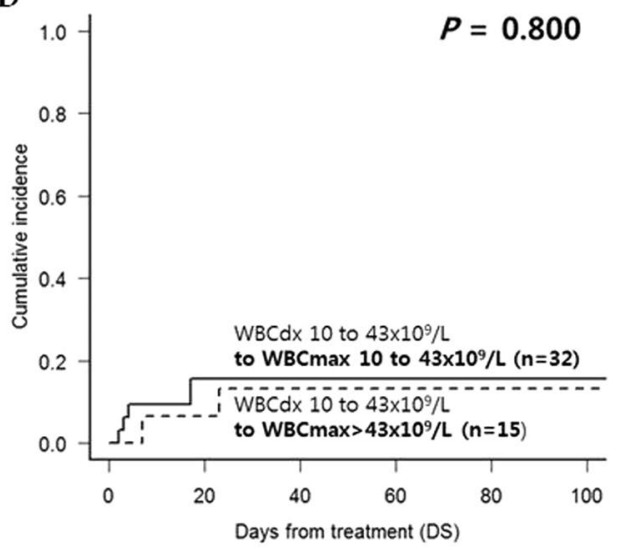

Figure 3. The different early outcomes according to leukocytosis progress. Subgroup analyses were performed in the group with WBCdx $<10 \times 10^{9} / \mathrm{L}$ (red bar [Lt.] and 3 red arrows in Fig. 2) and the group with WBCdx 10 to $43 \times 10^{9} / \mathrm{L}$ (blue bar [Lt.] and 2 blue arrows in Fig. 2). (A) Early death rates in the 3 subgroups with progression from WBCdx $<10 \times 10^{9} / \mathrm{L}$. (B) DS rates in the 3 subgroups with progression from WBCdx $<10 \times 10^{9} / \mathrm{L}$. (C) Early death rates in the 2 subgroups with progression from WBCdx 10 to $43 \times 10^{9} / \mathrm{L}$. (D) DS rates in the 2 subgroups with progression from WBCdx 10 to $43 \times 10^{9} / \mathrm{L}$.

correlated with early death or DS. Nonetheless, other recent studies have revealed that cell surface markers in APL blasts, such as CD34, CD56, T-cell antigen CD2, the BCR3 isoform, and the FLT3-ITD mutation are associated with hyperleukocytosis and a high risk of relapse $\mathrm{e}^{22}$. In addition, the presence of the FLT3-ITD mutation correlates with an increased occurrence of thrombotic events, increased leukocyte count, immature cell phenotypes such as CD34 and CD2, and the BCR3 isoform. Our data also showed a relationship between the FLT3 mutation and $B C R 3$ isoform, but in our data, WBCmax showing progressive leukocytosis was the most important independent factor for predicting early complications and long-term survival outcomes.

Although our current results are from a retrospective study, all enrolled patients were treated with the same management strategy for a long period, which could minimize possible biases. Unfortunately, because the standard treatment for APL has changed to a combination therapy using ATO, these results might no longer be applicable, so our results need further validation. In conclusion, our data show that a WBCmax indicating progressive leukocytosis correlated better with early death and DS than the Sanz-risk criteria. The role of dexamethasone prophylaxis, antithrombin III, and a cytoreduction strategy should be evaluated in specific patient subsets to reduce early events in APL. In addition, being in the high-increment WBCmax group also correlated with a high risk of relapse, so early intervention with close monitoring and preventive management might improve survival outcomes $^{23}$.

\section{Methods}

Study population. We initially identified 259 adult APL patients with a median age of 42 years (range; 15-72 years) from 2002 to 2014. We confirmed APL with a chromosomal analysis and additional PML-RARA reverse transcriptase polymerase chain reaction (RT-PCR), followed by reverse transcription quantitative PCR (RT-qPCR). To detect the karyotypes, at least 20 metaphases from BM cells were analyzed by the GTG banding method after 24 or $48 \mathrm{~h}$ of unsynchronized culture, and the International System for Cytogenetic Nomenclature was used as a guideline for classification ${ }^{24}$. We also identified the presence of additional chromosomal abnormalities. The purpose and experimental protocols of this research were approved by the Institutional Review 
A

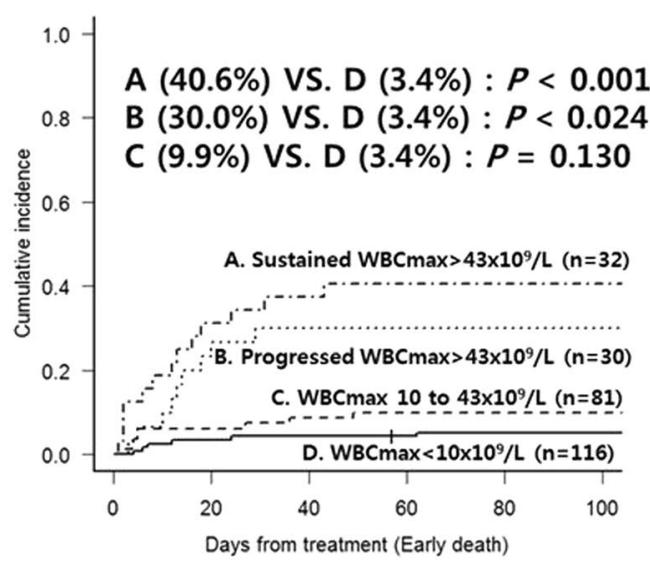

C

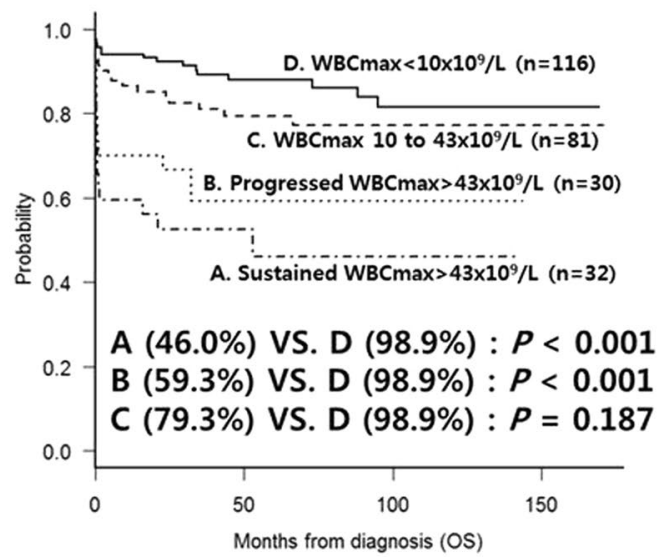

B

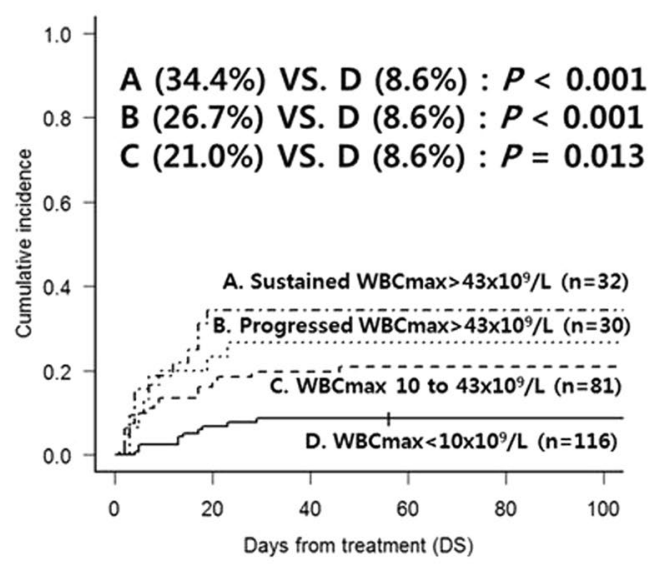

D

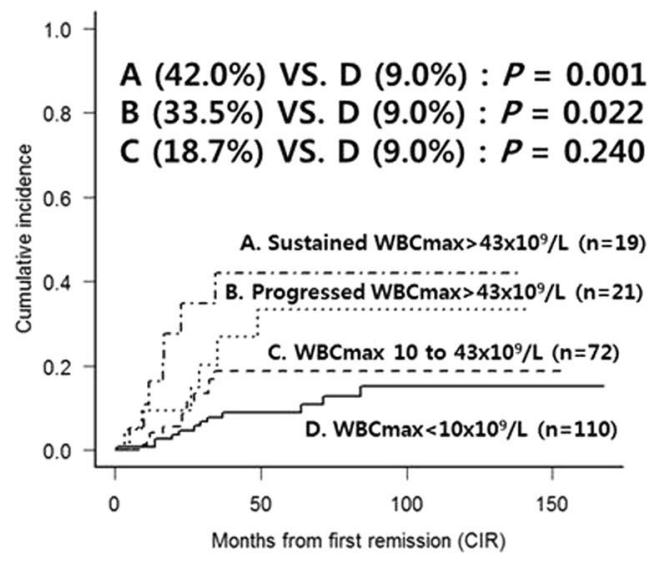

Figure 4. Survival outcomes of 4 subgroups stratified by the maximum leukocyte count (WBCmax) and progression of hyperleukocytosis. (A) Early death rates. (B) DS rates. (C) OS. (D) CIR.

Board and Ethics Committee guidelines of the Catholic Medical Center (KC15RISI0862) and the principles of the Declaration of Helsinki. Informed consent of all patients was obtained according to established guidelines. For patients younger than 18 years, consent was obtained from their legal guardians.

Treatment strategy and supportive care. Except for 1 woman, all patients received ATRA ( $45 \mathrm{mg}$ / $\mathrm{m}^{2} /$ day) divided into 2 daily doses immediately upon suspicion of APL or with morphological evidence from a BM aspiration study. We performed leukapheresis whenever leukocyte counts exceeded $50 \times 10^{9} / \mathrm{L}$, and some patients with hyperleukocytosis were treated with hydroxyurea, cytarabine, and prophylactic dexamethasone. Available blood products were vigorously administered with frequent monitoring. The target level for transfusion was higher than $50 \times 10^{9} / \mathrm{L}$ for platelets, higher than $120 \mathrm{mg} / \mathrm{dL}$ for fibrinogen, higher than $70 \%$ for prothrombin time, and higher than $70 \%$ for ATIII. Most patients were treated with induction chemotherapy using idarubicin $\left(12 \mathrm{mg} / \mathrm{m}^{2}\right.$, days $\left.1,3,5,7\right)$ based on the AIDA protocol, as previously reported ${ }^{1,18,19}$, but some patients $(\mathrm{n}=13)$ with serious infectious complications or comorbidity were treated with a compassionate program of ATO, and some $(\mathrm{n}=26)$ were treated with ATRA alone. After achievement of hematological CR, all patients received the same consolidation chemotherapy consisting of three courses in combination with 15 days of ATRA as follows - course 1 , idarubicin $\left(7 \mathrm{mg} / \mathrm{m}^{2}\right.$, days $\left.1-4\right)$; course 2 , mitoxantrone $\left(10 \mathrm{mg} / \mathrm{m}^{2}\right.$, days $\left.1-4\right)$; and course 3 , idarubicin $\left(12 \mathrm{mg} / \mathrm{m}^{2}\right.$, day $\left.1-2\right)$ - which was based on the LPA99 protocol from PETHEMA ${ }^{6,25}$. After completion of consolidation, patients received the following maintenance therapy for 2 years: 6-mercaptopurine $\left(50 \mathrm{mg} / \mathrm{m}^{2} /\right.$ day $)$ and ATRA for 15 days every 3 months. Our maintenance was a modified regimen (by exclusion of methotrexate) based on a protocol previously reported ${ }^{6,19,25}$. When DS occurred, we promptly discontinued ATRA and started dexamethasone (10 mg twice daily), with strict control of volume overloading using diuretics or renal replacement therapy.

Molecular studies. The molecular studies were performed from the BM samples collected at diagnosis, 1 month after induction and consolidation chemotherapies, and every 3 months after maintenance treatment. PML-RARA, the representative marker for measurable residual disease, was detected by a multiplex RT-PCR screening assay using a HemaVision kit (DNA Technology, Aarthus, Denmark), and quantification was performed using the RT-qPCR method (Real-Q PML-RARA quantification kit, Biosewoom, Korea) with a 


\begin{tabular}{|c|c|c|c|c|c|c|c|c|}
\hline \multirow[b]{3}{*}{ Variables } & \multicolumn{4}{|c|}{ Early death } & \multicolumn{4}{|c|}{ Differentiation syndrome (DS) } \\
\hline & \multicolumn{2}{|c|}{ Univariate } & \multicolumn{2}{|c|}{ Multivariate } & \multicolumn{2}{|c|}{ Univariate } & \multicolumn{2}{|c|}{ Multivariate } \\
\hline & \begin{tabular}{|l|} 
8-week \\
death
\end{tabular} & $p$ & $\begin{array}{l}\text { HR } \\
(95 \% \mathrm{CI})\end{array}$ & $p$ & $\begin{array}{l}\text { 8-week } \\
\text { DS }\end{array}$ & $p$ & \begin{tabular}{|l|} 
HR \\
$(95 \% \mathrm{CI})$
\end{tabular} & $p$ \\
\hline \multicolumn{9}{|l|}{ Age at diagnosis } \\
\hline$<40$ years $(\mathrm{n}=111)$ & $8.1 \%$ & $0.033 *$ & & & $18.2 \%$ & 0.933 & & \\
\hline$\geq 40$ years $(\mathrm{n}=148)$ & $17.6 \%$ & & $\begin{array}{l}2.38 \\
(1.1-5.1)\end{array}$ & $0.026^{*}$ & $17.6 \%$ & & & \\
\hline \multicolumn{9}{|l|}{ Platelet counts at diagnosis } \\
\hline$<30 \times 10^{9} / \mathrm{L}(\mathrm{n}=124)$ & $17.7 \%$ & 0.061 & & & $21.8 \%$ & 0.110 & & \\
\hline$\geq 30 \times 10^{9} / \mathrm{L}(\mathrm{n}=135)$ & $9.6 \%$ & & & & $14.2 \%$ & & & \\
\hline \multicolumn{9}{|l|}{ FLT3 mutation } \\
\hline No FLT3 mutation $(\mathrm{n}=118)$ & $6.8 \%$ & $0.006^{*}$ & & & $16.9 \%$ & 0.189 & & \\
\hline FLT3-ITD or -TKD $(\mathrm{n}=46)$ & $21.7 \%$ & & & & $26.1 \%$ & & & \\
\hline \multicolumn{9}{|l|}{ BCR subtype } \\
\hline BCR1 $(n=120)$ & $8.3 \%$ & 0.018* & & & $17.6 \%$ & 0.243 & & \\
\hline BCR3 $(\mathrm{n}=70)$ & $20.0 \%$ & & & & $24.3 \%$ & & & \\
\hline \multicolumn{9}{|l|}{ Leukocyte count at diagnosis } \\
\hline$<10.0 \times 10^{9} / \mathrm{L}(\mathrm{n}=180)$ & $8.3 \%$ & $<0.001^{*}$ & & & $15.6 \%$ & $0.026^{*}$ & & \\
\hline $10.0-43.0 \times 10^{9} / \mathrm{L}(\mathrm{n}=47)$ & $12.8 \%$ & & & & $14.9 \%$ & & & \\
\hline$>43.0 \times 10^{9} / \mathrm{L}(\mathrm{n}=32)$ & $40.6 \%$ & & & & $34.4 \%$ & & & \\
\hline \multicolumn{9}{|l|}{ Leukocyte count at peak (WBCmax) } \\
\hline$<10.0 \times 10^{9} / \mathrm{L}(\mathrm{n}=116)$ & $3.4 \%$ & $<0.001^{*}$ & 1.0 & & $8.6 \%$ & $0.001 *$ & 1.0 & \\
\hline $10.0-43.0 \times 10^{9} / \mathrm{L}(\mathrm{n}=81)$ & $9.9 \%$ & & $\begin{array}{l}1.12 \\
(0.3-3.4) \\
\end{array}$ & 0.843 & $21.0 \%$ & & $\begin{array}{l}3.06 \\
(1.4-6.6) \\
\end{array}$ & $0.004^{*}$ \\
\hline Progressed $>43.0 \times 10^{9} / \mathrm{L}(\mathrm{n}=30)$ & $30.0 \%$ & & $\begin{array}{l}3.72 \\
(1.4-9.8)\end{array}$ & $0.008^{*}$ & $26.7 \%$ & & $\begin{array}{l}3.29 \\
(1.3-8.2)\end{array}$ & $0.011^{*}$ \\
\hline Sustained $>43.0 \times 10^{9} / \mathrm{L}(\mathrm{n}=32)$ & $40.6 \%$ & & $\begin{array}{l}5.38 \\
(2.2-13.2)\end{array}$ & $<0.001 *$ & $34.4 \%$ & & $\begin{array}{l}5.79 \\
(2.5-13.4)\end{array}$ & $<0.001 *$ \\
\hline \multicolumn{9}{|l|}{ D-dimer at diagnosis } \\
\hline$\leq 20 \mathrm{mg} / \mathrm{L}(\mathrm{n}=132)$ & $3.0 \%$ & $<0.001^{*}$ & & & $15.2 \%$ & 0.238 & & \\
\hline$>20 \mathrm{mg} / \mathrm{L}(\mathrm{n}=127)$ & $24.4 \%$ & & $\begin{array}{l}6.39 \\
(2.2-18.4)\end{array}$ & $0.001 *$ & $20.6 \%$ & & & \\
\hline \multicolumn{9}{|l|}{ ATIII level at diagnosis } \\
\hline$\leq 100 \%(\mathrm{n}=191)$ & $16.8 \%$ & $0.011^{*}$ & & & $21.1 \%$ & $0.023^{*}$ & $\begin{array}{l}2.51 \\
(1.1-5.9)\end{array}$ & $0.036^{*}$ \\
\hline$>100 \%(\mathrm{n}=68)$ & $4.4 \%$ & & & & $8.8 \%$ & & & \\
\hline \multicolumn{9}{|l|}{ Fibrinogen level at diagnosis } \\
\hline$<140 \mathrm{mg} / \mathrm{dL}(\mathrm{n}=139)$ & $19.4 \%$ & $0.002 *$ & & & $20.1 \%$ & 0.300 & & \\
\hline$\geq 140 \mathrm{mg} / \mathrm{dL}(\mathrm{n}=120)$ & $6.7 \%$ & & & & $15.1 \%$ & & & \\
\hline
\end{tabular}

Table 2. Multivariate analysis of affecting factors for early death and differentiation syndrome. Abbreviation: CIR, cumulative incidence of relapse; HR, hazard ratio; FLT3, Fms-like tyrosine kinase 3; ITD, internal tandem duplication; TKD, tyrosine kinase domain; BCR, breakpoint cluster region; ATRA, all-trans retinoic acid; ATIII, Antithrombin III.

sensitivity of $5.0 \log \left(10^{-5}\right)$. The RQ-PCR level represented the ratios of $P M L-R A R A$ normalized to the expression of the reference gene, $A B L 1\left(1.0 \times 10^{4}\right)$. The FLT3 mutation was detected using multiplex allele-specific RT-PCR (ABSOULTE FLT3 TKD/ITD RT-PCR, Biosewoom, Korea). Because those molecular studies became available in 2006, we could not obtain molecular data for patients treated before that.

Primary endpoint and definitions. The primary endpoint of the current study was to determine predictive factors that affect early complications, early death, and DS, in APL patients. Therefore, we tried to identify the prognostic value of progressive hyperleukocytosis after ATRA administration during initial treatment. We checked the diagnostic level (WBCdx) and maximum level (WBCmax) of the leukocyte counts during the initial treatment period and identified subgroups with progressive hyperleukocytosis, i.e., those with a low WBCdx $<10 \times 10^{9} / \mathrm{L}$ whose WBCmax was significantly higher than that. The definition of early death was all patients who died within 8 weeks of treatment. DS was diagnosed as the presence of at least 2 of the following - dyspnea, unexplained fever, sudden weight gain greater than $5 \mathrm{~kg}$, unexplained hypotension, acute renal failure, or a chest radiograph demonstrating pulmonary infiltration or pleuropericardial effusion ${ }^{4,13}$. Any patient showing 4 or more of those features was considered to have severe DS, and those with fewer than 4 were classified as having moderate DS. 
Statistical analysis. All categorical variables were compared by Fisher's exact tests, and continuous variables were assessed with the Mann-Whitney $U$ test. To determine a significant cut-off level at which WBCmax could predict early death or DS, we used a ROC curve analysis. OS and DFS rates were calculated using Kaplan-Meier survival analyses, and the log-rank test was used to evaluate differences between subgroups. CIR and NRM rates were calculated using cumulative incidence estimations that treated non-relapse deaths and relapse as competing risks for CIR and NRM, respectively, and they were compared using the Gray test ${ }^{26}$. Early death and DS rates were also calculated by cumulative incidence estimations that used untreated patients and early deaths as competing risks, respectively. Multivariate analyses for the cumulative incidence of early death and DS were calculated using a Fine-Gray proportional hazard regression model. All statistical analyses were performed using ' $\mathrm{R}$ ' software (ver. 2.15.1, R Foundation for Statistical Computing, 2012). Statistical significance was set at a $p$ value $<0.05$.

\section{References}

1. Sanz, M. A. et al. A modified AIDA protocol with anthracycline-based consolidation results in high antileukemic efficacy and reduced toxicity in newly diagnosed PML/RARalpha-positive acute promyelocytic leukemia. PETHEMA group. Blood 94, 3015-3021 (1999).

2. Mandelli, F. et al. Treatment of elderly patients ( $>$ or $=60$ years) with newly diagnosed acute promyelocytic leukemia. Results of the Italian multicenter group GIMEMA with ATRA and idarubicin (AIDA) protocols. Leukemia 17, 1085-1090, https://doi. org/10.1038/sj.leu.2402932 (2003).

3. Grimwade, D. et al. Refinement of cytogenetic classification in acute myeloid leukemia: determination of prognostic significance of rare recurring chromosomal abnormalities among 5876 younger adult patients treated in the United Kingdom Medical Research Council trials. Blood 116, 354-365, https://doi.org/10.1182/blood-2009-11-254441 (2010).

4. Sanz, M. A. et al. Risk-adapted treatment of acute promyelocytic leukemia based on all-trans retinoic acid and anthracycline with addition of cytarabine in consolidation therapy for high-risk patients: further improvements in treatment outcome. Blood 115, 5137-5146, https://doi.org/10.1182/blood-2010-01-266007 (2010).

5. Ades, L. et al. Is cytarabine useful in the treatment of acute promyelocytic leukemia? Results of a randomized trial from the European Acute Promyelocytic Leukemia Group. J Clin Oncol 24, 5703-5710, https://doi.org/10.1200/JCO.2006.08.1596 (2006).

6. Sanz, M. A. et al. Risk-adapted treatment of acute promyelocytic leukemia with all-trans retinoic acid and anthracycline monochemotherapy: long-term outcome of the LPA 99 multicenter study by the PETHEMA Group. Blood 112, 3130-3134, https:// doi.org/10.1182/blood-2008-05-159632 (2008).

7. Lo-Coco, F. et al. Retinoic acid and arsenic trioxide for acute promyelocytic leukemia. N Engl J Med 369, 111-121, https://doi. org/10.1056/NEJMoa1300874 (2013).

8. Platzbecker, U. et al. Improved Outcomes With Retinoic Acid and Arsenic Trioxide Compared With Retinoic Acid and Chemotherapy in Non-High-Risk Acute Promyelocytic Leukemia: Final Results of the Randomized Italian-German APL0406 Trial. J Clin Oncol 35, 605-612, https://doi.org/10.1200/JCO.2016.67.1982 (2017).

9. Efficace, F. et al. Randomized phase III trial of retinoic acid and arsenic trioxide versus retinoic acid and chemotherapy in patients with acute promyelocytic leukemia: health-related quality-of-life outcomes. J Clin Oncol 32, 3406-3412, https://doi.org/10.1200/ JCO.2014.55.3453 (2014).

10. Abaza, Y. et al. Long-term outcome of acute promyelocytic leukemia treated with all-trans-retinoic acid, arsenic trioxide, and gemtuzumab. Blood 129, 1275-1283, https://doi.org/10.1182/blood-2016-09-736686 (2017).

11. Cicconi, L. \& Lo-Coco, F. Current management of newly diagnosed acute promyelocytic leukemia. Ann Oncol 27, 1474-1481, https://doi.org/10.1093/annonc/mdw171 (2016).

12. De Botton, S. et al. Incidence, clinical features, and outcome of all trans-retinoic acid syndrome in 413 cases of newly diagnosed acute promyelocytic leukemia. The European APL Group. Blood 92, 2712-2718 (1998).

13. Montesinos, P. et al. Differentiation syndrome in patients with acute promyelocytic leukemia treated with all-trans retinoic acid and anthracycline chemotherapy: characteristics, outcome, and prognostic factors. Blood 113, 775-783, https://doi.org/10.1182/ blood-2008-07-168617 (2009).

14. Rahme, R. et al. Early death in acute promyelocytic leukemia (APL) in French centers: a multicenter study in 399 patients. Leukemia 28, 2422-2424, https://doi.org/10.1038/leu.2014.240 (2014).

15. Park, J. H. et al. Early death rate in acute promyelocytic leukemia remains high despite all-trans retinoic acid. Blood 118, 1248-1254, https://doi.org/10.1182/blood-2011-04-346437 (2011).

16. Vahdat, L. et al. Early mortality and the retinoic acid syndrome in acute promyelocytic leukemia: impact of leukocytosis, low-dose chemotherapy, PMN/RAR-alpha isoform, and CD13 expression in patients treated with all-trans retinoic acid. Blood 84, 3843-3849 (1994).

17. de la Serna, J. et al. Causes and prognostic factors of remission induction failure in patients with acute promyelocytic leukemia treated with all-trans retinoic acid and idarubicin. Blood 111, 3395-3402, https://doi.org/10.1182/blood-2007-07-100669 (2008).

18. Mandelli, F. et al. Molecular remission in PML/RAR alpha-positive acute promyelocytic leukemia by combined all-trans retinoic acid and idarubicin (AIDA) therapy. Gruppo Italiano-Malattie Ematologiche Maligne dell'Adulto and Associazione Italiana di Ematologia ed Oncologia Pediatrica Cooperative Groups. Blood 90, 1014-1021 (1997).

19. Lee, S. et al. The significance of minimal residual disease kinetics in adults with newly diagnosed PML-RARalpha-positive acute promyelocytic leukemia: results of a prospective trial. Haematologica 91, 671-674 (2006).

20. Sanz, M. A. et al. Definition of relapse risk and role of nonanthracycline drugs for consolidation in patients with acute promyelocytic leukemia: a joint study of the PETHEMA and GIMEMA cooperative groups. Blood 96, 1247-1253 (2000).

21. Norsworthy, K. J. \& Altman, J. K. Optimal treatment strategies for high-risk acute promyelocytic leukemia. Curr Opin Hematol 23, 127-136, https://doi.org/10.1097/MOH.0000000000000215 (2016).

22. Testa, U. \& Lo-Coco, F. Prognostic factors in acute promyelocytic leukemia: strategies to define high-risk patients. Ann Hematol 95, 673-680, https://doi.org/10.1007/s00277-016-2622-1 (2016).

23. Yoon, J. H. et al. High WT1 expression is an early predictor for relapse in patients with acute promyelocytic leukemia in first remission with negative PML-RARa after anthracycline-based chemotherapy: a single-center cohort study. J Hematol Oncol 10, 30, https://doi.org/10.1186/s13045-017-0404-4 (2017).

24. Shaffer, L. G., Slovak, M. L., Campbell, L. J. \& Krager, E. S. ISCN An international system for human cytogenetic nomenclature. (1st ed. Ag, S. K., Basel, Switzerland) 138, 978-3805589857 (2009).

25. Sanz, M. A. et al. Risk-adapted treatment of acute promyelocytic leukemia with all-trans-retinoic acid and anthracycline monochemotherapy: a multicenter study by the PETHEMA group. Blood 103, 1237-1243, https://doi.org/10.1182/blood-2003-07-2462 (2004).

26. Gray, R. J. A class of k-sample tests for comparing the cumulative incidence of a competing risk. Annals of Statistics 16, 1141-1154 (1988). 


\title{
Acknowledgements
}

This study was supported by Research Fund of Seoul St. Mary's Hospital, The Catholic University of Korea, and also supported by a grant from the Basic Science Research Program through the National Research Foundation of Korea (NRF) funded by the Ministry of Education (2015R1D1A1A01059819).

\section{Author Contributions}

J.-H.Y. carried out the molecular research, collected and analyzed data, and wrote the manuscript; G.J.M., S.S.P., Y.-W.J., S.-E.L., B.-S.C., K.-S.E., Y.-J.K., S.L., C.-K.M., S.-G.C. and J.-W.L., H-J.K. designed and conducted the study, provided patients and materials, analyzed data, and wrote the manuscript; and all authors gave final approval of the manuscript.

\section{Additional Information}

Competing Interests: The authors declare no competing interests.

Publisher's note: Springer Nature remains neutral with regard to jurisdictional claims in published maps and institutional affiliations.

\begin{abstract}
(c) (i) Open Access This article is licensed under a Creative Commons Attribution 4.0 International License, which permits use, sharing, adaptation, distribution and reproduction in any medium or format, as long as you give appropriate credit to the original author(s) and the source, provide a link to the Creative Commons license, and indicate if changes were made. The images or other third party material in this article are included in the article's Creative Commons license, unless indicated otherwise in a credit line to the material. If material is not included in the article's Creative Commons license and your intended use is not permitted by statutory regulation or exceeds the permitted use, you will need to obtain permission directly from the copyright holder. To view a copy of this license, visit http://creativecommons.org/licenses/by/4.0/.
\end{abstract}

(c) The Author(s) 2019 